\title{
WIKIPEDIA AS DOMAIN KNOWLEDGE NETWORKS: Domain Extraction and Statistical Measurement
}

\author{
Zheng Fang, Jie Wang, Benyuan Liu \\ Department of Computer Science, University of Massachusetts, Lowell, MA, USA \\ zfang@cs.uml.edu,wang@cs.uml.edu,bliu@cs.uml.edu \\ Weibo Gong \\ Department of Electrical and Computer Engineering, University of Massachusetts, Amherst, MA, USA \\ gong@ece.umass.edu
}

\begin{abstract}
Keywords: Knowledge Network, Statistics, Power Law, Degree Distribution, Betweenness Centrality, Clustering Coefficient, Wikipedia, MathWorld

Abstract: $\quad$ This paper investigates knowledge networks of specific domains extracted from Wikipedia and performs statistical measurements to selected domains. In particular, we first present an efficient method to extract a specific domain knowledge network from Wikipedia. We then extract four domain networks on, respectively, mathematics, physics, biology, and chemistry. We compare the mathematics domain network extracted from Wikipedia with MathWorld, the web's most extensive mathematical resource created and maintained by professional mathematicians, and show that they are statistically similar to each other. This indicates that MathWorld and Wikipedia's mathematics domain knowledge share a similar internal structure. Such information may be useful for investigating knowledge networks.
\end{abstract}

\section{INTRODUCTION}

Wikipedia, created a decade ago, has developed and evolved rapidly into the most comprehensive online encyclopedia. While people enjoy using it, their concerns about the credibility of Wikipedia as an accurate knowledge source linger on, which has triggered extensive research in recent years.

A body of knowledge may be viewed as a network, where nodes in the network represent concepts and tools, and edges between nodes represent the relationship between them. The measurement of matureness of a specific knowledge network should include measures of accuracy and completeness. However, there has been no efficient algorithm to evaluate these two essential properties. A viable alternative would be to compare statistical properties of the target knowledge network with a trusted knowledge source, such as paper-based encyclopedia. Restricted by weight and volume, however, paper-based encyclopedias cannot be truly comprehensive for measuring completeness.

MathWorld, the web's most extensive mathematical resource created and maintained by professional mathematicians, matches the scale of Wikipedia's domain on mathematics and provides an excellent trusted source as a base for comparison with the mathematics knowledge network contained in Wikipedia. To carry out the comparison we must first extract the mathematics network contained in Wikipedia. In particular, we will need to devise an effective mechanism to extract a knowledge network in a specific domain from Wikipedia. To this end we devise a three-step method to extract a specific knowledge network from Wikipedia with high quality. We use this method to extract four domain knowledge networks from Wikipedia on, respectively, mathematics, physics, biology, and chemistry. We will refer to these networks as, respectively, Wikipedia Math, Wikipedia Physics, Wikipedia Biology, and Wikipedia Chemistry. We then carry out statistical analysis on these four knowledge networks.

We show that Wikipedia Math and MathWorld have similar statistical properties, which suggest that the MathWorld and Wikipedia Math share a similar internal structure. Such information may be served as supporting evidence and necessary condition for Wikipedia Math to be mature. While the comparison is on the mathematics domain, it still provides a strong indication that other domains in Wikipedia 
would also share such similarity with corresponding professionally maintained sources, for the contributors of Wikipedia are volunteers from all areas with no particular quality preference on the mathematics domain. The measurement of statistical similarity may be useful for investigating knowledge networks in addition to directly measuring accuracy and completeness.

The rest of the paper is organized as follows. Section 2 briefly reviews previous work on measuring and analyzing Wikipedia. Section 3 presents a three-step method to extract from Wikipedia a specific domain knowledge network, and compares Wikipedia Math with MathWorld. Section 4 concludes the paper.

\section{RELATED WORK}

Early attempts to measure Wikipedia, started in 2004, were focused on content accuracy and reputation (Lih, 2004; Giles, 2005; Chesney, 2006). Most of these attempts were centered on the metrics of content correctness and quality, not on the completeness of the topics covered. For example, Voss (Voss, 2005) performed statistical analysis on all available language versions of Wikipedia. He examined a number of articles and the growth rate in each available language version of Wikipedia. Halavais and Lackaff (Halavais and Lackaff, 2008) analyzed the diversity of the content of Wikipedia. They selected a number of topics in paper-based encyclopedia and matched them with those in Wikipedia. They concluded that Wikipedia is indeed diversified in its content coverage.

Other work has focused on the relationship between collaborative editing and content quality (Zlatić et al., 2006; Kittur and Kraut, 2008). These studies indicated that in the online collaboration environment, the quality of the content can be improved through cooperation of multiple contributors over time, and coordination can lead to better quality. However, there has been no direct evidence provided to measure the quality of the content currently presented in Wikipedia.

While most of the research treats Wikipedia as one complex network, a number of authors have investigated the hierarchical structure of Wikipedia. For example, Muchnik et al (Muchnik et al., 2007) studied how to automatically discover hierarchies in Wikipedia, Yu et al (Yu et al., 2007) studied how to evaluate ontology with Wikipedia categories, and Silva et al (Silva et al., 2010) studied how to identify borders of certain knowledge networks.

Previous analysis of degree distributions in Wikipedia were focused on the entire Wikipedia, which concluded that the Wikipedia's degree distribution follows the power law, the same as that of the World Wide Web (Voss, 2005; Capocci, 2006; Kamps and Koolen, 2009).

\section{STATISTICAL MEASUREMENT OF WIKIPEDIA}

In the case when there exists a trusted mature knowledge network of a certain domain, measuring the same domain knowledge networks extracted from Wikipedia can be carried out by comparing the topic coverage and the statistical structures of the two networks. The growth of online resources has provided trusted domain-specific knowledge that could match the scale of Wikipedia in the same domain. For example, MathWorld, produced by Wolfram Research, is a reliable and extensive resource on the knowledge of mathematics. It was created and has been maintained by a group of mathematicians and related professionals for over a decade, and is therefore viewed as a mature knowledge network on mathematics. The existence of MathWorld allows us to compare the matureness of Wikipedia.

\subsection{Extraction of a Specific Domain Knowledge Network}

The richness of content presented in Wikipedia, while being the reason for the popularity of Wikipedia, also makes it difficult to extract a specific knowledge domain, for the boundaries between domains are blurry as people keep pushing the fringes and adding more materials.

To extract a certain domain knowledge network from Wikipedia, e.g chemistry, an ideal approach would be to find a complete list of unambiguous chemistry terminologies (this list should contain words of all named reactions and named compounds, among other things), extract all Wikipedia pages whose titles match the words in the list, and rebuild links between these pages. Commonly used chemistry dictionaries would help, but not sufficient, since the completeness and unambiguity are hardly guaranteed (e.g. mercury as a chemical element vs. mercury as a planet).

Page tracing is an effective alternative to building the chemistry knowledge network from Wikipedia. That is, starting from the main chemistry page, titled "Chemistry", we follow all the links presented in this page to include new pages to the network, and do so recursively until no new pages are found. Note that it 
is quite often that a link from a page in the domain of chemistry will lead to a page in a completely different domain. Therefore, appropriate filters should be applied to examine the links to ensure that the linked pages are still in the scope of chemistry.

Filters may be a keyword-based pass filter or a keyword-based block filter. Given a set of predetermined keywords in a specific domain, a pass filter passes a page if the page title matches or contains one of these keywords, and blocks it otherwise. Given a set of pre-determined keywords not in the given domain, a block filter blocks a page if the title of the page matches or contains one of the keywords, and passes it otherwise. Constructing either type of filters, however, is challenging, for it is difficulty to predict what kind of keywords will appear or not appear in the title of a page and the outgoing link could point to a completely different and unexpected category. In particular, an overstrict pass filter may fail to include pages that should be included, while an insufficient block filter may include pages that should not be included. There is another major drawback in page tracing: It may fail to discover pages that are not reachable from other pages. Such "hidden" pages may be newly created pages that have not been linked to from any existing page, pages that contain fringe topics known to only a small number of people, or pages that consist of rarely used organic compounds in chemistry.

To overcome these drawbacks, we devise an effective method to extract a domain knowledge network from Wikipedia based on page titles and page categories. The page category, a feature of Wikipedia, is used to classify pages. Each page belongs to one or more categories and each category contains several pages or sub-categories. The categories of a page can be found at the end of the page, and the category itself can be viewed as a special page. Although typically hidden from the user, the category information can be obtained by explicitly loading the page with the "Category:" prefix. We use page categories to generate new pages and check whether a page should be included in the network. Our extraction mechanism consists of the following three steps.

\section{Step 1. Extract Domain Category Hierarchy}

Each category may contain a number of pages or subcategories, thus categories themselves could form a hierarchy, representing a framework of the domain knowledge. We use page categories to generate new pages in this step.

An ideal domain hierarchy should be a directed acyclic graph containing exactly those nodes in the domain, but the Wikipedia category hierarchies are far from being ideal. In particular, a category hierarchy may contain two types of loops. They are local loops and out-domain loops. By local loops it means that two or more closely related categories (sometimes they are actually the same but with slightly different categorical descriptions) contain each other. This type of loops has no important effect on the domain knowledge generation. By out-domain loops it means loops containing a node in a different domain. Out-domain loops could be catastrophic if not handled properly, for they might lead to a super-category that contains chemistry as a subcategory. Take the following outdomain loop as an example:

$$
\begin{aligned}
& \text { Chemistry } \rightarrow \cdots \rightarrow \text { Silicon } \rightarrow \cdots \rightarrow \text { Memory } \rightarrow \\
& \text { Knowledge } \rightarrow \text { Science } \rightarrow \cdots \rightarrow \text { Chemistry }
\end{aligned}
$$

where the ambiguity of "Memory" leads to a misinterpretation as human memory instead of computer memory as it is intended to be, which in turn leads to the super-category of "Knowledge" of human knowledge. If such out-domain loop is not handled properly, all categories under "Knowledge" would be included. Fortunately, such out-domain loops are rare, as the category misclassification errors are reported by users everyday, and Wikipedia editors correct such errors efficiently.

Deploying keyword-based block filters can avoid such misinterpretations by properly selecting a set of keywords. We repeat the process of generating the domain category hierarchy several times and update the block filter accordingly until a satisfactory quality is obtained.

\section{Step 2. Extract Pages}

With the hierarchy of domain categories at hand, we are ready to extract pages that belong to the hierarchy. We note that some pages listed under a category in the hierarchy may not belong to the domain of interest and should not be included. For example, a biography of a chemist might be listed in the hierarchy and should not be included in the Wikipedia Chemistry network. The category information of a page can be used to check for inclusion. Since a page might belong to several different categories, the page which should not be included would contain keyword in the titles of its concatenated categories. For instance, chemists also belong to the category of "People". To avoid adding chemists into our knowledge network, we can simply add a keyword "People" to the block filter. Other similar block-keywords would be "Birth", "Prize", and "Facility", to name only a few. 
After extracting pages that pass the block filter, we scan each page for linkage and build links within the current set of pages. No new pages will be included at this point.

Step 3. Trim Disconnected Component

Note that certain pages in the network after the previous two steps might belong to a cluster that are disconnected from any other node in the network. This can be used to remove further unwanted pages that are still contained in the category hierarchy. Intuitively, the misclassified categories will generate its own pages that clustered together, but with no links to the target domain. For example, the "metal music genre", which is misclassified as a subcategory of "metal" and passes the block filter, will generate pages such as "history of metal music", "metal music band", "songs", and other things. But none of these pages has links to the chemistry knowledge network. Removing the largest connected component of such pages, we will have a network of higher quality with less noise.

Remark. Theoretically it may be possible to have disconnected components that contain the wanted domain knowledge. But such phenomena never happened in our experiments. This makes sense, for the real-world domain knowledge networks probably do not work that way. It is hard to imagine that within the same domain there are parts that are completely isolated from each other. We also note that, although the data set we extracted from Wikipedia may still contain misclassified nodes, the fraction of these nodes is very small. Thus, any negative effect there is to the accuracy of statistical analysis will be small and can be safely ignored.

We present four domain knowledge networks extracted from Wikipedia using our three-step method. They are Wikipedia Math, Wikipedia Physics, Wikipedia Biology, and Wikipedia Chemistry. Table 1 shows the number of nodes and edges for each of these networks.

Table 1: Wikipedia four domains statistics.

\begin{tabular}{c|c|c|c|c}
\hline & Math & Phys & Bio & Chem \\
\hline \hline Nodes & 18,673 & 16,132 & 162,726 & 39,674 \\
\hline Edges & 295,772 & 206,878 & $2,526,351$ & $1,677,833$ \\
\hline
\end{tabular}

\subsection{Comparison of Wikipedia Math with MathWorld}

We compare Wikipedia Math with MathWorld on degree distribution, betweenness centrality, and clustering coefficients. Jia et al (Jia and Guo, 2009) provided the statistical analysis of MathWorld, which will serve as the base of our comparison.

\subsubsection{Degree Distribution}

The MathWorld network studied in (Jia and Guo, 2009) contains over 12,000 entries as on December 1st, 2008 (MathWorld currently contains 13,067 entries as on May 3rd, 2011). The degree distributions of MathWorld and Wikipedia Math are plotted in Figure 1 .

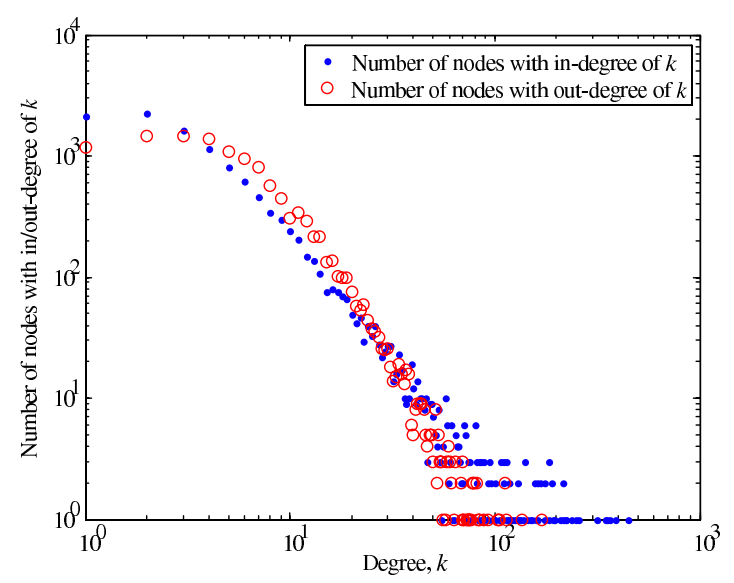

(a) MathWorld (Jia and Guo, 2009)

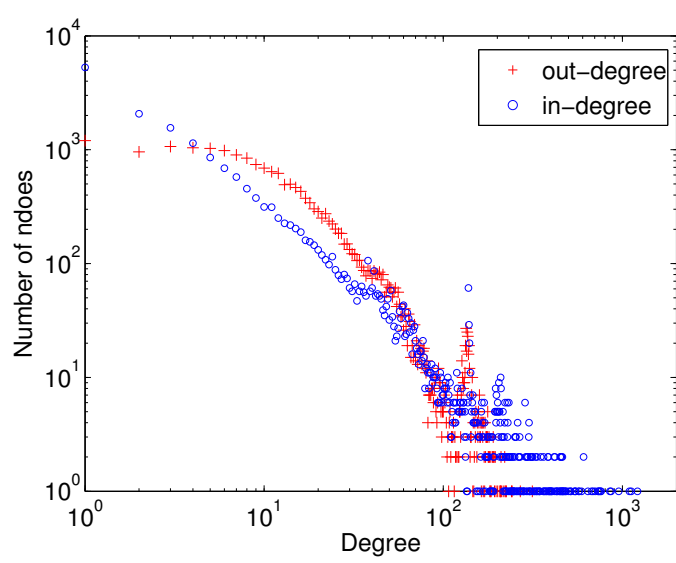

(b) Wikipedia Math

Figure 1: Degree distribution of MathWorld and Wikipedia Math

It can be seen from the degree plots that both indegree and out-degree distributions between Math- 
World and Wikipedia Math are very similar, and exhibits power-law behavior. The data run short of the heavy tail and drop down quickly at the end. This phenomenon also happens in some other studies on the power law, which is analyzed and discussed in (Gong et al., 2005). In the Wikipedia Math plot, the vertical feature around degree 100 implies that the data set contains several ensemble pages (a.k.a. directory pages) that serve as containers of a list of pages. For instance, "outline of probability" and "NP-complete problems" are container pages.

\subsubsection{Betweenness Centrality}

Betweenness centrality of node $i$ is defined by

$$
b c(i)=\sum_{j, k \in N, i \neq j \neq k} \frac{n_{j k}(i)}{n_{j k}}
$$

where $n_{j k}$ denotes the number of shortest paths from node $j$ to node $k$ and $n_{j k}(i)$ is the number of presence of $i$ on the shortest paths. A node will have a large betweenness centrality value if it connects a large number of nodes on their shortest paths. In a knowledge network, the nodes of large betweenness centrality would be considered as important fundamental concepts in the knowledge network. The comparison of betweenness centrality is shown in Figure 2.

Most nodes in MathWorld and Wikipedia Math have low betweenness centrality with a value less than 1. Only a small portion of nodes have very large betweenness centrality values. Table 2 lists top ten highest betweenness centrality topics in both MathWorld (Jia and Guo, 2009), Wikipedia Math, Wikipedia Physics, Wikipedia Biology, and Wikipedia Chemistry. The top ten topics in Wikipedia Math approximately match those in MathWorld. This suggests a similar set of core knowledge that play an important role in connecting knowledge are shared in both MathWorld and Wikipedia Math. The topics with top 10 betweenness centrality in the rest of the domains also match important concepts of each domain.

\subsubsection{Clustering Coefficient}

The clustering coefficient of a node is a measure of direct connectivity of its neighbors. There is evidence suggests that in most real-world networks, especially in social networks, nodes tend to create tightly knit groups over those in randomly generated networks (Holland and Leinhardt, 1971; Watts and Strogatz, 1998). Therefore, the clustering coefficient may be used to discover hierarchies in a given network (Watts and Strogatz, 1998). The measurement

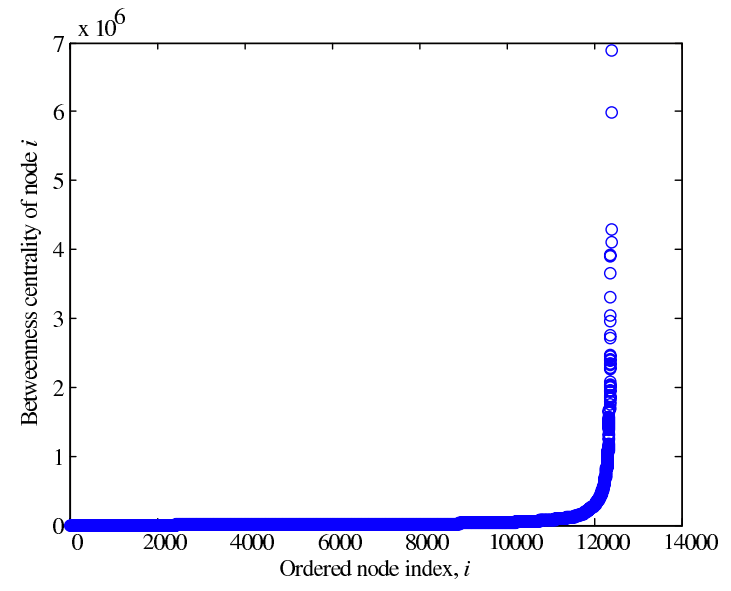

(a) MathWorld (Jia and Guo, 2009)

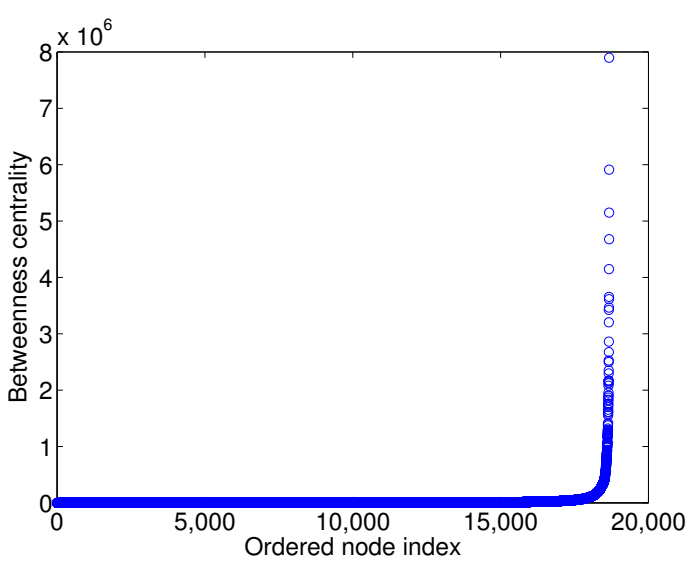

(b) Wikipedia Math

Figure 2: Betweenness centrality of MathWorld and Wikipedia Math

of clustering coefficient for node $i$ in a directed graph is defined as follows (Caldarelli, 2007):

$$
\begin{aligned}
C_{i}^{\text {in }} & =\frac{1}{k_{i}^{\text {in }}\left(k_{i}^{\text {in }}-1\right)} \sum_{j, k} A_{j i} A_{k i} \frac{A_{j k}+A_{k j}}{2} \\
C_{i}^{\text {out }} & =\frac{1}{k_{i}^{\text {out }}\left(k_{i}^{\text {out }}-1\right)} \sum_{j, k} A_{i j} A_{i k} \frac{A_{j k}+A_{k j}}{2}
\end{aligned}
$$

Where $k_{i}^{\text {in }}$ and $k_{i}^{\text {out }}$ denote, respectively, the in-degree and out-degree of node $i$, and $A_{i j}$ equals 1 if there exists an edge from $i$ to $j, 0$ otherwise.

The clustering coefficients plotted against ordered node indexes is shown in Figure 3. In MathWorld, the average in-clustering and out-clustering coefficients reported are 0.2536 and 0.1980 (Jia and Guo, 2009), respectively, while in Wikipedia Math the corresponding numbers are 0.2324 and 0.2898 . The inclustering coefficient is similar but the out-clustering coefficient is slightly higher in Wikipedia Math. This 
Table 2: Top 10 topics with highest betweenness centrality

\begin{tabular}{c|c|c|c|c|c}
\hline & MathWorld & Math & Physics & Biology & Chemistry \\
\hline \hline 1 & Circle & Statistics & Physics & Animal & Carbon \\
\hline 2 & Polynomial & Integer & Hydrogen & Plant & Hydrogen \\
\hline 3 & Binomial Coefficient & Function & Refractive & Insect & Chemical Compound \\
\hline 4 & Prime Number & Topology & Quantum Mechanics & Arthropod & NMR Spectroscopy \\
\hline 5 & Integer & Matrix & Molecule & Fungus & Catalysis \\
\hline 6 & Set & Group & Density & Gene & Infrared Spectroscopy \\
\hline 7 & Matrix & Vector Space & Wavelength & Protein & Chemical Reaction \\
\hline 8 & Group & Number Theory & General Relativity & Enzyme & Chemistry \\
\hline 9 & Power & Prime Number & Temperature & Lepidoptera & Relative Permittivity \\
\hline 10 & Graph & Set & Magnetic Field & Bacteria & Nucleotide \\
\hline
\end{tabular}

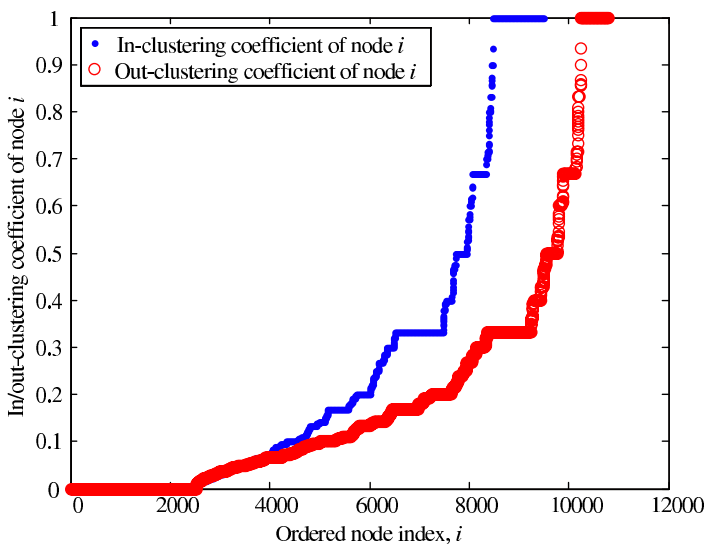

(a) MathWorld (Jia and Guo, 2009)

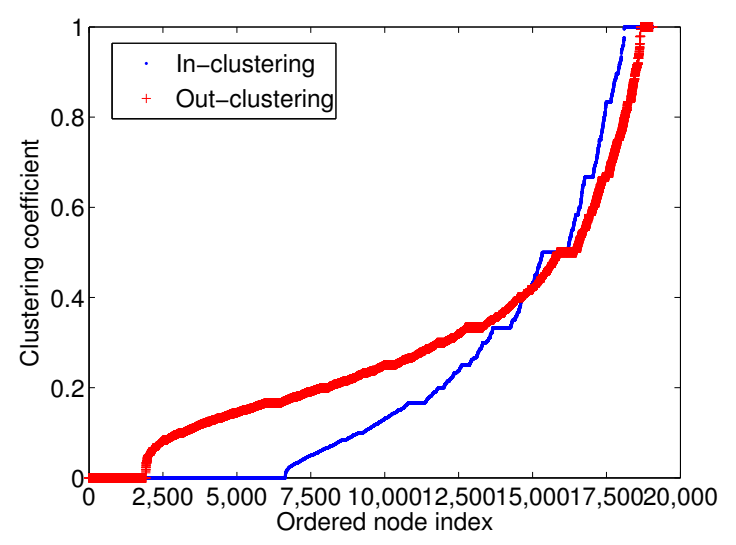

(b) Wikipedia Math

Figure 3: Clustering coefficient in MathWorld and Wikipedia Math

can be explained as follows: In Wikipedia Math, topics are often elaborated in more details with more references (i.e., with more outgoing links) to fundamental concepts used in the articles. Compare with the theoretical value $O\left(|N|^{-1}\right)$ in random networks $\left(8.3 \times 10^{-5}\right.$ for MathWorld and $5.4 \times 10^{-5}$ for Wikipedia Math), the average clustering coefficients in two mathematics networks are both substantially larger by several order of magnitude, which indicate that both networks contain a hierarchical structure.

\subsection{From Mathematics to Other Domains}

The comparison results between MathWorld and Wikipedia Math suggest that these two mathematical knowledge networks are statistically similar. This matches the common impression of knowledge in Wikipedia's mathematical domain. Such statistical similarity suggests that MathWorld and Wikipedia Math share a similar internal structure, which may be served as supporting evidence and necessary condition to measure the matureness of Wikipedia Math.

It would be more comprehensive to compare other domain knowledge networks with corresponding professionally maintained sources of the same domain as well. However, such sources rarely exist and if any, is hard to obtain. Nevertheless, although our comparison is only based on the domain of mathematics, it still provides a strong indication that other domains in Wikipedia would also share the statistical similarity with corresponding professionally maintained sources, for the contributors of Wikipedia are from all areas and have no preference of particular quality on the domain of mathematics.

\section{CONCLUSIONS}

Measuring Wikipedia and studying its properties as a comprehensive human knowledge network have attracted much attention in knowledge discovery and complex system. In this paper we compare Wikipedia 
Math with MathWorld from a statistical point of view, and conclude that Wikipedia Math is statistically similar to MathWorld.

To compare Wikipedia's domain knowledge networks, we devise an efficient three-step method to extract domain knowledge with high quality. Four domain knowledge networks on mathematics, physics, biology, and chemistry are extracted from Wikipedia. Our experiments demonstrate the effectiveness of the proposed method. Statistical analysis are carried out on the four domains knowledge networks.

\section{ACKNOWLEDGEMENTS}

The first author was supported in part by the NSF under grant CCF-0830314. The second author was supported in part by the NSF under grants CCF-0830314, CNS-0958477, and CNS-1018422. The third author was supported in part by the NSF under grants CNS0721626, CNS-0953620, and CNS-1018303. The fourth author was supported in part by NSF under grant EFRI-0735974 and by the Army Research Office under Contract W911NF-08-1-0233.

The authors thank $\mathrm{Xu}$ Yuan for his help on the early stage of knowledge network extractions.

\section{REFERENCES}

Caldarelli, G. (2007). Scale-Free Network. Oxford Univeristy Press.

Capocci, A. (2006). Preferential attachment in the growth of social networks: The internet encyclopedia wikipedia". Phys. Rev. E; Physical Review E, 74(3).

Chesney, T. (2006). An empirical examination of wikipedia's credibility. Firstmonday, 11.

Giles, J. (2005). Internet encyclopaedias go head to head. Nature, 438(7070):900-901.

Gong, W., Liu, Y., Misra, V., and Towsley, D. F. (2005). Self-similarity and long range dependence on the internet: a second look at the evidence, origins and implications. Computer Networks, 48(3):377-399.

Halavais, A. and Lackaff, D. (2008). An analysis of topical coverage of wikipedia. Journal of ComputerMediated Communication, 13(2):429-440.

Holland, P. W. and Leinhardt, S. (1971). Transitivity in structural models of small groups. Comparative Group Studies, 2(2):107-124.

Jia, Q. and Guo, Y. (2009). Discovering the knowledge hierarchy of mathworld for web intelligence. In Fuzzy Systems and Knowledge Discovery, 2009. FSKD '09. Sixth International Conference on, volume 7, pages $535-539$.
Kamps, J. and Koolen, M. (2009). Is wikipedia link structure different? In Proceedings of the Second ACM International Conference on Web Search and Data Mining, WSDM '09, pages 232-241, New York, NY, USA. ACM.

Kittur, A. and Kraut, R. E. (2008). Harnessing the wisdom of crowds in wikipedia: quality through coordination. In Proceedings of the 2008 ACM conference on Computer supported cooperative work, CSCW '08, pages 37-46, New York, NY, USA. ACM.

Lih, A. (2004). Wikipedia as participatory journalism: Reliable sources? metrics for evaluating collaborative media as a news resource. Proceedings of the International Symposium on Online Journalism 2004.

Muchnik, L., Itzhack, R., Solomon, S., and Louzoun, Y. (2007). Self-emergence of knowledge trees: Extraction of the wikipedia hierarchies. Physical Review E (Statistical, Nonlinear, and Soft Matter Physics), $76(1)$.

Silva, F. N., Travencolo, B. A., Viana, M. P., and da Fontoura Costa, L. (2010). Identifying the borders of mathematical knowledge. Journal of Physics A: Methematical and Theoretical, 43(325202).

Voss, J. (2005). Measuring wikipedia. In Proceedings of the 10th International Conference of the International Society for Scientometrics and Informetrics.

Watts, D. and Strogatz, S. (1998). Collective dynamics of small-world networks. Nature, 393(6684):440-442.

Yu, J., Thom, J. A., and Tam, A. (2007). Ontology evaluation using wikipedia categories for browsing. In Proceedings of the sixteenth ACM conference on Conference on information and knowledge management, CIKM '07, pages 223-232, New York, NY, USA. ACM.

Zlatić, V., Božičević, M., Štefančić, H., and Domazet, M. (2006). Wikipedias: Collaborative web-based encyclopedias as complex networks. Physical Review E, 74(1):016115. 